\title{
Caracterização dos Núcleos de Apoio à Atenção Básica - Saúde Mental de uma região de saúde
}

\section{Characterization of the Primary Care Support Centers - Mental Health in a health region}

\author{
Guilherme Emanuel Weiss Pinheiro, Luciane Prado Kantorski
}

\section{Como citar este artigo:} PINHEIRO, CUILHERME E. U.; KANTORSKI, LUCIANE P. Caracterização dos Núcleos de Apoio à Atenção Básica - Saúde Mental de uma região de saúde. Revista Saúde (Sta. Maria). 2020; 46 (2).

\section{Autor correspondente:}

Nome: Guitherme Emanuel W. Pinheiro E-mail: enfermeiro.guipinheiro@gmail.com Telefone: (51) 9 8016-7020

Formação Profissional: Enfermeiro e Doutor em Ciências pela Universidade Federal de Pelotas (UFPel), Pelotas, RS, Brasil, Mestre em Ensino na Saúde pela

Universidade Federal de Ciências da Saúde de Porto Alegre (UFCSPA), Porto Alegre, RS, Brasil.

Filiação Institucional: Colégio Politécnico da Universidade Federal de Santa Maria Endereço para correspondência: Av. Roraima, $n^{\circ}: 1000$ Bairro: Camobi

Cidade: Santa Maria Estado: Rio Grande do Sul CEP: 97 105-900

Data de Submissão:

09/06/2020

Data de aceite: 17/09/2020

Conflito de Interesse: Não há conflito de interesse

\section{(cc) $\mathrm{B} Y-\mathrm{NC}-\mathrm{ND}$}

\section{RESUMO}

Objetivo: caracterizar o apoio matricial em uma região de saúde do Rio Grande do Sul, a partir dos núcleos de apoio à atenção básica - saúde mental. Métodos: pesquisa qualitativa, realizada com núcleos de apoio à atenção básica de três municípios de uma região de saúde do estado do Rio Grande do Sul, os quais mantinham o referido núcleo. A coleta de dados ocorreu nos meses de novembro e dezembro de 2018, de forma online com profissionais de apoio matricial, os quais preencheram formulário eletrônico sobre as características do município, do núcleo e do trabalho do apoio matricial. A pesquisa recebeu aprovação de Comitê de Ética em Pesquisa. Resultados: são apresentadas as composições da rede de atenção à saúde, a composição e a caracterização dos núcleos de apoio à atenção básica da região e a organização do trabalho dos núcleos, em especial, na revisão da prática de encaminhamentos. Considerações Finais: o trabalho do apoio matricial, viabilizado pelos núcleos de apoio à atenção básica, propicia oferta de serviços e responsabilização à atenção básica, por meio das ações de educação permanente em saúde, cuidado compartilhado e supervisão em municípios de pequeno porte, buscando favorecer a oferta de ações de saúde mental às pessoas em sofrimento psíquico no território.

PALAVRAS-CHAVE: Saúde mental; Atenção primária à saúde; Assistência integral à saúde; Equipe de assistência ao paciente.

\section{ABSTRACT}

Objective: characterize matrix support in a health region of Rio Grande do Sul, from the support center to primary care - mental health. Methods: qualitative research, carried out with support centers for primary care in three municipalities in a health region in the state of Rio Grande do Sul, which maintained the said center. Data collection took place in the months of November and December 2018, online with matrix support professionals, who filled out an electronic form on the characteristics of the municipality, the nucleus and the work of matrix support. The research received approval from the Research Ethics Committee. Results: the compositions of the health care network are presented, the composition and characterization of the support centers for primary care in the region and the organization of the work of the centers, especially in the review of the referral practice. Final Considerations: the matrix support work, made possible by the primary care support centers, provides the provision of services and accountability to primary care, through the actions of permanent health education, shared care and supervision in small municipalities, seeking to favor offering mental health actions to people in psychological distress in the territory.

KEYWORDS: Mental health; Primary health care; Comprehensive health care; Patient care team. 


\section{INTRODUÇÃO}

O Brasil sofreu uma mudança radical no campo da saúde mental, posto que se efetivou a substituição de um paradigma hospitalocêntrico para um modelo de serviços de base comunitária, atrelado aos territórios, com ações intersetoriais, tendo como princípios a inclusão social e a autonomia das pessoas. Esse processo veio acompanhado de mudanças nas políticas de saúde, surgindo dessa reforma, novos serviços que transformaram as ações no campo da saúde, colocando a pessoa com transtorno mental no centro do processo de cuidado¹.

A partir disso, o movimento de luta antimanicomial tomou forma e muitas conquistas foram construídas ao longo desses anos. Entre as conquistas destaca-se a Atenção Básica (AB) como componente da Rede de Atenção Psicossocial (RAPS), mantendo responsabilidades e competência para assistir as situações de saúde mental comuns e recorrentes no território².

$\mathrm{A} A \mathrm{~B}$ conta com 0 apoio matricial como uma forma de realizar a atenção à saúde de maneira compartilhada com o objetivo de prestar cuidado integral, resolutivo e interdisciplinar ${ }^{3,4}$. Assim, o apoio matricial é uma estratégia que ampara os profissionais, as pessoas com transtorno mental, as famílias e as comunidades no cuidado, na promoção e na reabilitação da saúde ${ }^{5}$.

Para que o apoio matricial atue de forma resolutiva, há a necessidade de construções de relações dialógicas entre os atores envolvidos, a partir da lógica interprofissional e da prática colaborativa, buscando a integralidade do cuidado ${ }^{6}$. Dessa forma que o apoio matricial em saúde mental na AB busca a integração dos profissionais especialistas, chamados de apoiadores, com profissionais das equipes de referência, denominados apoiados, por meio de práticas interdisciplinares que auxiliam na ampliação da clínica, da qualidade da assistência e na resolutividade dos serviços ${ }^{7}$.

No ano de 2008, foram instituídos no Brasil os Núcleo de Apoio à Saúde da Família (NASF), com o objetivo de garantir maior integração e prestar apoio às equipes da Estratégia Saúde da Família $(E S F)^{8}$, fomentando a criação de núcleos de apoio matricial. Essa proposta não contemplou a grande maioria dos municípios brasileiros devido a critérios populacionais, porém foi um progresso no que diz respeito à qualificação das práticas e transformação do modelo de atenção à saúde.

No Rio Grande do Sul, em 2011, foi criado um serviço no campo da saúde mental, chamado Núcleo de Apoio à Atenção Básica - Saúde Mental (NAAB) ${ }^{9}$, pois não havia previsão na política nacional de serviços de apoio matricial para municípios de pequeno porte, realidade de aproximadamente $80 \%$ dos municípios gaúchos ${ }^{10}$. No ano seguinte, 0 governo federal ampliou os critérios de implantação de NASF ${ }^{11}$, modificando critérios e universalizando o acesso dos municípios a esse serviço.

Assim, o NAAB previa a formação de um núcleo profissional com dois profissionais de nível superior e um de nível médio, com carga horária mínima de 20 horas semanais, coincidente com o horário de trabalho das equipes de ESF, 
com financiamento para cada núcleo. Além disso, os profissionais deveriam possuir diferentes formações, construindo parcerias com as ESF por meio de ações de apoio matricial, do compartilhamento de ações de promoção em saúde nos territórios e da construção da rede intersetorial ${ }^{9}$.

Como prerrogativa legal, constava a responsabilização compartilhada entre as equipes de $A B$ e a equipe do NAAB, prevendo ações para viabilização da revisão da prática do encaminhamento com base nos processos de referência e contra referência ${ }^{9}$. No ano de 2012, haviam 99 equipes de NAAB no estado ${ }^{12}$ e em 2015 o número era de 118 equipes ${ }^{13}$.

Com isso, ressalta-se a importância em caracterizar esses núcleos, que são recentes, a fim de conhecer a composição das equipes, 0 atendimento às questões elencadas na legislação e as ações que realizam nos territórios. Este artigo tem como objetivo caracterizar o apoio matricial em uma região de saúde do Rio Grande do Sul, a partir do Núcleos de Apoio à Atenção Básica - Saúde Mental.

\section{MÉTODOS}

Trata-se de pesquisa de abordagem qualitativa, realizada de forma online com profissionais dos NAABs de uma região de saúde do estado do Rio Grande do Sul, com três municípios que mantinham o núcleo no período de coleta. Constituindo-se como recorte de uma pesquisa de doutorado sobre avaliação de experiências de apoio matricial, com base na proposta de avaliação de quarta geração ${ }^{14}$. A diretriz COREQ (Consolidated Criteria for Reporting Qualitative Research) foi utilizada para orientar a redação deste artigo.

A coleta de dados ocorreu por meio do preenchimento de informações em formulário eletrônico via Google Forms $®$, nos meses de novembro e dezembro de 2018. As atividades de pesquisa foram conduzidas pelos autores deste artigo, que possuem formação na área da enfermagem, além de experiência em estudos qualitativos e em avaliação de serviços. Buscou-se a compreensão descritiva em relação aos núcleos implantados na região. A escolha da região foi intencional e se deu pelo fato do primeiro autor deste artigo manter vínculo profissional com uma universidade que mantém projetos de ensino, pesquisa e extensão com os municípios adjacentes ao da sede da instituição, sendo que a região de saúde escolhida é a que possui maior número de NAABs entre as regiões próximas. Os critérios de seleção dos participantes foram: atuar em município da região escolhida e que mantém NAAB na rede; ser profissional atuante junto ao NAAB; maior de 18 anos; aceitar participar da pesquisa, por meio do termo de consentimento livre e esclarecido.

A região estudada pertence a área de abrangência da $4^{\text {a }}$ Coordenadoria Regional de Saúde - Santa Maria, localizada na região central do estado do Rio Grande do Sul, especificamente a Região de Saude 2 - Entre Rios, composta por onze municípios e com população estimada de 123.022 habitantes ${ }^{10}$. Segundo relatórios do Ministério da Saúde a região tem uma cobertura de AB de 89,14\% e de ESF de 83,26\%, com 33 equipes de ESF e 198 agentes comunitários de saúde em atividade ${ }^{15}$. 
Os participantes foram os profissionais, membros das equipes dos NAABs, sendo eles: psicólogos(as) (3); terapeutas ocupacionais (3); artesã (1); e acompanhante terapêutica (1). Não houve recusa para participação no estudo. Os municípios foram identificados pela palavra "Município" seguida de número arábico. 0 instrumento de coleta foi composto por questões sobre: composição da rede de ateção à saúde, implantação do NAAB, projeto, constituição, forma de contratação, carga horária, atuação dos núcleos, processo de trabalho, legislação de criação do núcleo, gerenciamento de recursos, monitoramento e avaliação. Após o preenchimento do formulário pelas equipes, os dados foram analisados pelo Método Comparativo Constante ${ }^{14}$, considerando a legislação que cria o NAAB ${ }^{9}$.

Os aspectos éticos foram contemplados em todos os momentos deste estudo, conforme prevê a Resolução № 466/2012 do Conselho Nacional de Saúde ${ }^{16}$ sobre Pesquisa com Seres Humanos. A pesquisa foi submetida à apreciação a um Comitê de Ética em Pesquisa (CEP), recebendo sua aprovação sob parecer № 3.038.987, obtendo Certificado de apresentação para Apreciação Ética (CAAE), sob o número: 02237118.2.0000.5316. Os participantes aceitaram os termos do Termo de Consentimento Livre e Esclarecido por meio do preenchimento do questionário online.

\section{RESULTADOS}

Os resultados encontrados dizem respeito à composição da Rede de Atenção à Saúde (RAS) - Tabela 01, à caracterização do NAABs como ano de implantação, composição, carga horária, forma de contratação e ações - Tabela 02 e o trabalho dos núcleos no sentido de revisar a prática de encaminhamentos. Em relação à RAS dos municípios todos possuem: equipes da ESF, Hospital Geral e Centro de Referência em Assistência Social (CRAS). Ainda, cada município mantém outros dispositivos conforme a tabela 1.

Tabela 01. Caracterização da Rede de Atenção à Saúde de uma região de saúde, Rio Grande do Sul, 2019.

\begin{tabular}{llll}
\hline Características / Município & Município 1 & Município 2 & Município 3 \\
\hline População $^{10}$ & 12.561 & 10.848 & 8.721 \\
N $^{0}$ Dispositivos da RAS & 9 & 8 & 6 \\
Oficina Terapêutica & $\operatorname{Sim}$ & $\operatorname{Sim}$ & Não \\
NASF & $\operatorname{Sim}$ & Não & Sim \\
Leito de Saúde Mental em Hospital Geral & $\operatorname{Sim}$ & Não & Não \\
\hline
\end{tabular}

Fonte: elaboração própria.

No que diz respeito às composições das equipes dos NAABs, dentre os profissionais de nível superior, todos contam com um profissional de psicologia e um profissional de terapia ocupacional. Apenas um núcleo mantém profissional 
de nível médio, conforme tabela 2. Sobre o projeto de implantação do NAAB, as equipes referem conhecê-lo em sua totalidade ou em parte, ainda, afirmam ter conhecimento sobre o documento que cria o NAAB na Política Estadual de Saúde Mental.

Tabela 02. Caracterização dos Núcleo de Apoio à Atenção Básica de uma região de saúde, Rio Grande do Sul, 2019

\begin{tabular}{llll}
\hline Características / Município & Município 1 & Município 2 & Município 3 \\
\hline Ano de implantação & 2012 & 2012 & 2014 \\
Equipe completa & Sim & Não & Não \\
Vínculo dos profissionais & Contrato temporário & Estatutário & Contrato temporário \\
Carga horária & $>60$ horas & $<60$ horas & $>60$ horas \\
Manteve mesma equipe & Não & Em parte & Sim \\
Ações de revisão da prática & Acompanhamentos & Ações intersetoriais & Visitas domiciliares, \\
de encaminhamento & em saúde mental, & e internações em & referência para os \\
& discussão de casos, & saúde mental & atendimentos da AB, \\
& visitas domiciliares, & & discussão de casos \\
& ações educativas, & & e atendimentos \\
& avaliações e & & interdisciplinares \\
& encaminhamentos & & \\
\hline
\end{tabular}

Fonte: elaboração própria.

Sobre o trabalho dos NAABs, realizam ações diretamente com as pessoas com transtornos mentais, em colaboração às equipes de $A B$, atuando na lógica da linha de cuidado, acolhimento, vínculo e acompanhamento longitudinal de corresponsabilidade das equipes. A natureza dessas ações está voltada para a revisão da prática de encaminhamento. Os profissionais apontaram que realizam discussões de casos e atendimentos compartilhados com as equipes de $A B$ e referem articular com a rede de saúde, com a rede intersetorial, realizando atendimentos e visitas domiciliares, sendo esses atendimentos tanto conjuntos, quanto individuais, ou até mesmo em grupos. No que diz respeito às ações de Educação Permanente em Saúde (EPS), todos os núcleos relatam participar com outras equipes de apoio (tanto em nível central, como em nível regional), além disso, promovem ações de EPS com equipes de AB.

\section{DISCUSSÕES}

A discussão dos resultados foi dividida em três eixos, o primeiro diz respeito às RAS dos municípios, o segundo sobre a composição e as características dos núcleos de apoio matricial e o terceiro discute as o trabalho do apoio matricial na região. 


\section{Redes de Atenção à Saúde}

Atualmente uma das preocupações tem sido a inclusão das ações de saúde mental na AB. Dessa forma, o apoio matricial atua com a possibilidade de trabalhar na lógica das redes e com estratégias de comunicação entre os profissionais dos serviços, aumentando a oferta de possibilidades de cuidado às pessoas no sistema de saúde ${ }^{17}$.

AAB é a ordenadora do cuidado na comunidade e contribui como articuladora do cuidado em saúde mental. Nesse sentido, o Brasil tem como estratégia prioritária à ESF, que é considerada como dispositivo da RAPS e tem construído resultados positivos no cuidado às pessoas com transtornos mentais, por meio de ações no território, articuladas com a rede e a partir das diversas dimensões das pessoas ${ }^{18}$.

Resultados de um estudo, realizado com apoiadores e enfermeiros no sul do Brasil, apontam que há potencialidades nas ações de apoio matricial em saúde mental na $A B$ e convergem para a materialização do cuidado compartilhado, transformador de individualidades e do coletivo. Embora sejam vivenciados desafios na construção desta rede, o apoio matricial é uma ferramenta de trabalho que contribui na aproximação do campo da saúde mental com a RAS, aproximando profissionais, serviços e pessoas com transtornos psíquicos ${ }^{19}$. Este panorama colabora para que a rede, tendo a $\mathrm{AB}$ como coordenadora do cuidado, construa alternativas associadas ao apoio matricial no campo da saúde mental.

O trabalho em rede tem como intenção atuar na efetivação da garantia do direitos das pessoas a uma assistência integral à saúde, necessitando ser visto de forma ampliada e contextualizada, devido a isso se faz interessante compreender que a rede de saúde é composta por outros dispositivos, além daqueles habitualmente compreendidos como as unidades de saúde, hospitais, serviços especializados, incorporando as escolas, os espaços de lazer, os dispositivos de assistência social, entre outros. Na tentativa de ampliar esta visão é que a RAS se fortalece e que as ações de saúde mental na $A B$ começam a se constituir e se configurar como um importante espaço para construção de novos modelos de cuidado ${ }^{6}$.

Corroborando a isso, um estudo, realizado no nordeste brasileiro com profissionais de saúde mental e da $A B$ destaca que o apoio matricial tem se apresentado como o organizador do fluxo das demandas e tem promovido a integração entre os serviços da rede de atenção à saúde. Ainda, destaca-se como articulador, ampliando a oferta de possibilidades e promovendo um itinerário terapêutico às pessoas em sofrimento mental nos serviços, com enfoque na $A B^{20}$.

Observando a composição das RAS nos municípios estudados é possível destacar a importância que a diversidade de dispositivos, além de sua articulação e construção conjunta, possuem para o cuidado em saúde mental. Além do mais, a AB, porta de entrada preferencial do sistema, considerada ordenadora dos fluxos e dos cuidados, precisa contar com outros serviços, formando uma rede ampla e abrangente, para assim contribuir para o cuidado em saúde mental no território. 


\section{Composição e características do Apoio Matricial}

Todas as equipes caracterizadas são compostas por profissionais de nível superior, psicologia e terapia ocupacional, e apenas uma conta com profissionais de nível médio. São profissões que não constam no arcabouço legal como equipes mínimas de ESF, mas encontram-se elencadas nas legislações de instituição do NASF $^{11}$ e do NAAB ${ }^{9}$. É importante destacar que o apoio matricial não é campo do saber de determinada profissão ou formação, mas sim é a associação de diferentes visões e uma construção coletiva diante do trabalho em saúde.

A psicologia vem transformando sua prática ao longo dos últimos anos, buscando apropriar-se de outros conhecimentos, priorizando o olhar integral sobre as pessoas, suas famílias e comunidades, buscando romper com a visão dicotomizada entre clínico e social. Dessa forma, está construindo elementos que buscam agregar outros saberes em seu fazer cotidiano ${ }^{21}$.

Associada a psicologia, a terapia ocupacional no campo apoio matricial tende a contribuir com o saber de núcleo. Destarte, a prática interdisciplinar, a construção coletiva e as intervenções compartilhadas foram destaques em uma revisão sobre o trabalho desta profissão no apoio matricial, dialogando com outros saberes profissionais. Uma das principais contribuições se concentra na potencialização de intervenções a partir das necessidades das pessoas, compreendendo o cotidiano e as estruturas extra-muros dos serviços de saúde como espaço de cuidado ${ }^{22}$.

Os profissionais de nível médio atuantes no apoio matricial disparam ações juntos às pessoas acompanhadas, atuando lado-a-lado aos demais profissionais, contribuindo com um olhar diferenciado, o qual não vem carregado de uma visão clínica ou institucional, mas com a visão de expertise, a partir da experiência e da vivência. Dessa forma, fazem a diferença no cotidiano dos serviços que compõem a rede. Nota-se que o estado do Rio Grande do Sul prioriza a inserção destes profissionais nos núcleos ${ }^{9}$, diferente do NASF que prevê somente profissionais de nível superior ${ }^{11}$.

A contratação dos profissionais é considerada um nó crítico na discussão acerca dos núcleos de apoio matricial. Nesse sentido, verifica-se que apenas um município mantém profissionais de carreira no NAAB, nos demais são contratos temporários, o que se caracteriza como vínculo frágil. Essa forma de recrutamento está mais suscetível à mudanças na equipe e rotatividade dos profissionais, o que é possível constatar, pois apenas um município manteve a mesma equipe desde a implantação.

Estudo com profissionais de núcleos de apoio matricial, realizando em uma capital brasileira, aponta que há diferentes tipos de vínculo entre os profissionais e demonstra que inclusive os gestores acessam os cargos por meio de vínculos frágeis ${ }^{23}$. Outro estudo afirma que é possível identificar quando um município prioriza e aposta no apoio matricial, um dos indicativos pode ser a forma de ingresso dos profissionais. Esta precisa se dar a partir de concursos públicos, com seleções específicas para o apoio matricial e com exigência de experiência na área ${ }^{24}$. 
Os profissionais do apoio matricial e das equipes de ESF de uma região de saúde do nordeste brasileiro apresentam como limitação do trabalho a precarização dos vínculos, pois a maioria dos trabalhadores possuem contratos temporários ${ }^{25}$. As formas precárias de contrato e vínculo dos profissionais do apoio matricial preponderam em um estudo de caso com municípios do sudeste ${ }^{26}$. Já outro estudo, realizado com profissionais do apoio matricial e com gestores no centro-oeste brasileiro, demonstrou vinculação estável por meio de concurso público, além de valorização da remuneração dos trabalhadores, reforçando que vínculos fortes e remuneração adequada contribuem para o desenvolvimento melhor do trabalho ${ }^{27}$.

Outro elemento apresentado na caracterização diz respeito a carga horária dos núcleos, como é possível observar, apenas um município mantém o preconizado na normativa, mínimo 60 horas semanais. A reduzida carga horária dedicada ao apoio matricial e, ao mesmo tempo, o elevado número de equipes apoiadas, podem ser considerados como pontos frágeis e empecilhos para que o matriciamento se desenvolva de forma a responder as necessidades do território ${ }^{24}$. Um estudo que analisou a integração entre o apoio matricial e as equipes de referência, na região metropolitana de uma capital do sul do Brasil, aponta a demanda excessiva, o deslocamento dos profissionais para o desenvolvimento de ações e a dificuldade de trabalhar na lógica do apoio matricial, como os principais motivos para que a carga horária não seja suficiente ${ }^{28}$.

\section{O trabalho dos NAABS}

O NAAB tem no seu rol de atribuições ser motivador da revisão da prática de encaminhamentos. O que promove a ampliação da prática para um processo pactuado de linha de cuidado, caracterizado pela facilitação dos percursos da pessoa com transtorno psíquico pela rede, priorizando o acolhimento, o vínculo e o acompanhamento longitudinal. Dessa forma, inserindo ações de cuidado em saúde mental na AB por meio de discussão de casos, de visitas domiciliares, de realização de grupos, ações de EPS e demais intervenções.

A revisão desta prática está alicerçada na concepção de trabalho em rede, comparada a uma teia com várias conexões. E o apoio matricial é uma ferramenta que articula os cuidados em saúde mental, de forma colaborativa às equipes de ESF no território ${ }^{5}$. Estudo realizado no sul do Brasil percebe que as ações de saúde mental no território estão alinhadas as motivações dos profissionais, uma vez que entre suas intenções e expectativas estão os cuidados às pessoas com transtornos psíquicos, suas famílias e comunidades ${ }^{29}$. Este processo motivador promove o debate e leva a revisões das práticas de encaminhamentos para uma prática interprofissional e colaborativa.

As dimensões didático-pedagógico e assistencial do apoio matricial propiciam o debate juntamente aos serviços da $\operatorname{RAS}^{30}$. Construindo a partir disso, estratégias de cuidado às pessoas por meio de intervenções e ações no território e de suporte às equipes de ESF, alternativas às práticas de encaminhamentos para uma visão coletiva do trabalho. 
É importante pontuar que as ações em saúde mental na $A B$ não se restringem a apenas um serviço ou equipe, mas a práticas precisam ocorrer de forma articulada, em rede e de maneira colaborativa no território. Para que estas ações se constituam de forma efetiva são necessárias ações educativas e de qualificação dos trabalhadores neste contexto6. Assim, as intervenções didático-pedagógicas atuam para articular estes conhecimentos, a fim de habilitar e empoderar profissionais para desenvolver a escuta qualificada e atenta, além de ações de acolhimento das demandas de saúde mental ${ }^{17}$. Ainda é preciso avançar para a superação da lógica de encaminhamentos, prevendo a utilização de outros níveis de atenção com racionalidade, valorizando a $A B$ como nível responsável e capaz de dar conta da maioria das situações de saúde do território.

As ações ocorrem de diferentes formas, sejam elas ações educativas junto às equipes de referência do território ou ações intersetoriais com serviços que compõem a rede, ambas com o objetivo de qualificar a atenção e a assistência à saúde, em especial à saúde mental. 0 cuidado compartilhado, a construção coletiva e colaborativa são marcas do trabalho dos núcleos estudados, o que reflete em processos de cuidado interprofissionais que impactam diretamente no cotidiano dos serviços e na integralidade do cuidado.

\section{CONSIDERAÇÕES FINAIS}

Ao caracterizar os NAABs de uma região de saúde é possível afirmar que o trabalho do apoio matricial, viabilizado por este disposto, propicia serviços e responsabilização à $A B$, por meio das ações de EPS, cuidado compartilhado e supervisão em municípios de pequeno porte, buscando favorecer as ações de saúde mental às pessoas em sofrimento psíquico no território.

Embora este estudo tenha englobado a totalidade de municípios que mantém o NAAB em uma região de saúde, há a limitação de que os resultados retratam uma realidade específica. Entretanto, oferece pistas para a articulação do apoio matricial em saúde mental na $A B$, uma vez que discute características das RAS, das equipes de NAAB, aspectos relacionados ao trabalho, de superação da prática de encaminhamentos e de qualificação das equipes apoiadas, o que pode contribuir para reflexões em outros contextos.

Com isso, o presente artigo oferece subsídios para a manutenção deste núcleo na política de saúde mental, uma vez que atua na lógica do apoio aos demais dispositivos da rede e trabalham fortalecendo a AB. Assim, os NAABs buscam se consolidar como ferramenta interdisciplinar de trabalho em saúde e de cuidado em saúde mental no território.

\section{AGRADECIMENTOS}

O presente trabalho foi realizado com apoio da Coordenação de Aperfeiçoamento de Pessoal de Nível Superior - Brasil (CAPES) - Código de Financiamento 001. 


\section{REFERÊNCIAS}

1. Delgado PGGThe challenge of production of knowledge on the Brazilian psychiatric reform process. Cien Saude Colet. 2015;20(2):312-312. doi: 10.1590/1413-81232015202.17692014.

2. Ministério da Justiça (Brasil). Rede de Atenção Psicossocial no SUS: Eixo Políticas e Fundamentos [Internet]. 2018 [cited 2020 Jun 2]. Available from: http://www.aberta.senad.gov.br/medias/original/201704/20170424-094953-001.pdf

3. Ministério da Saúde (Brasil). HumanizaSUS: equipes de referência e apoio matricial. Brasília: Ministério da Saúde; 2004. 16 p.

4. Campos GWDS, Domitti AC. Apoio matricial e equipe de referência: uma metodologia para gestão do trabalho interdisciplinar em saúde. Cad Saude Pública. 2007;23(2):399-407. doi: 10.1590/S0102-311X2007000200016.

5. Nascimento MGG, Nadaleti NP, Vilela S de C, Terra F de S, Silva SA da, Resck ZMR. Nursing work process in the promotion of mental health: reflective analysis. Rev enferm Cent-Oeste Min. 2017;7:e2097. doi: 10.19175/recom.v7i0.2097.

6. Souza ÂC de, Amarante PD, Abrahão AL. Inclusion of mental health in primary health care: care strategy in the territory. Rev bras enferm. 2019;72(6):1677-82. doi: 10.1590/0034-7167-2018-0806.

7. Almeida DR, Soares JNC, Dias MG, Rocha FC, Neto GR de A, Andrade DLB. Care for carriers of mental disorder in primary care: an interdisciplinary and multiprofessional practice. Rev Pesqui (Univ Fed Estado Rio J, Online). 2020;12:420-5. doi: 10.9789/2175-5361.rpcfo.v12.8388.

8. Ministério da Saúde (Brasil). Núcleo de Apoio à Saúde da Família. Brasília: Ministério da Saúde; 2014. 118 p.

9. Comissão Intergestores Bipartite (Rio Grande do Sul). Resolução no 403/11 - CIB/RS, de 26 de outubro de 2011. Cria os Núcleos de Apoio à Atenção Básica (NAAB) - saúde mental, dentro da Política Estadual da Atenção Básica. Diário Oficial do Estado do Rio Grande do Sul, 03 Nov 2011.

10. Instituto Brasileiro de Geografia e Estatística. IBGE Cidades [Internet]. homepage. 2019 [cited 2019 Jun 28]. Available from: https://cidades.ibge.gov.br/ 
11. Ministério da Saúde (Brasil). Portaria n 3.124, de 28 de dezembro de 2012. Redefine os parâmetros de vinculação dos Núcleos de Apoio à Saúde da Família (NASF) Modalidades 1 e 2 às Equipes Saúde da Família e/ou Equipes de Atenção Básica para populações específicas, cria a Modalidade NASF 3, e dá outras providências. Diário Oficial da República Federativa do Brasil, 31 dez. 2012. Seção 1, p. 223.

12. Zanardo GL de P. Reinternações psiquiátricas: Análise das características sociodemográficas, clínicas e do uso da rede de atenção psicossocial de usuários com transtornos mentais [dissertação]. [Porto Alegre]: Pontifícia Universidade Católica do Rio Grande do Sul; 2016. 83 p.

13. Simoni ACR, Siqueira E, Soares J, Cabral KV, Adamy PE, Charão RB, Heinzelmann R, Almeida SA. Percursos da redução de danos no Rio Grande do Sul. Saúde Redes. 2015;1(1):57-65. doi: 10.18310/2446-4813.2015v1n1p57-65.

14. Guba EG, Lincoln YS. Avaliação de quarta geração. Campinas: Editora UNICAMP; 2011.

15. Ministério da Saúde (Brasil). e-Gestor AB - Informação e Gestão da Atenção Básica [Internet]. 2020 [cited 2020 Jun 2]. Available from: https://egestorab.saude.gov.br/paginas/acessoPublico/relatorios/relHistoricoCobertura.xhtml

16. Conselho Nacional de Saúde (Brasil). Resolução n 466, de 12 de dezembro de 2012. Aprovar as diretrizes e normas regulamentadoras de pesquisas envolvendo seres humanos. Diário Oficial da República Federativa do Brasil, 13 jun. 2013. Seção 1, p. 59.

17. Tatmatsu DB, Araújo ACC. Atenção Primária e Saúde Mental: Contribuições e Potencialidades do Apoio Matricial. Mudanças. 2016;24(2):71-9. doi: 10.15603/2176-1019/mud.v24n2p71-79.

18. Costa EVS, Cunha MC, Carvalho ME de, Negreiros JA, Oliveira EN, Neto FRGX. Salud Mental en Atención Primaria: taller de herramientas de enfoque familiar. Cult. cuid. 2018;(51):133-43.

19. Oliveira GC de, Schneider JF, Pinho LB de, Camatta MW, Nasi C, GuimarãesAN, Torres MEL. Matrix support actions in Primary Health Care: a phenomenological study. Acta Paul Enferm. 2019;32(6):674-82. doi: 10.1590/1982-0194201900093.

20. Silva SM da, Silva AM da, Souza AR De, Moura ADA, Lima GG De, Feitoza AR. Family health strategy: mental health care actions. Rev enferm UERJ. 2017;25: e16926. doi: 10.12957/reuerj.2017.16926. 
21. Cela M, Oliveira IF de. O psicólogo no Núcleo de Apoio à saúde da Família: articulação de saberes e ações. Estud Psicol. 2015;20(1):31-9. doi: 10.5935/1678-4669.20150005.

22. Jacinto B de O, Rodrigues $C$ de S, Maxta BSB, Tomasi ARP. $O$ apoio matricial em saúde realizado por terapeutas ocupacionais no Sistema Único de Saúde. Cad Ter Ocup UFSCar. 2017;25(1):191-201.doi: 10.4322/0104-4931.ctoAR0772.

23. Reis ML, Medeiros M, Pacheco LR, Caixeta CC. Evaluation of the multiprofessional work of the Family Health Support Center (NASF). Texto \& contexto enferm. 2016;25(1): e2810014. doi: 10.1590/0104-070720160002810014.

24. Castro CP de, Oliveira MM de, Campos GW de S. Matrix Support in the SUS of Campinas: how an inter-professional practice has developed and consolidated in the health network. Cien Saude Colet. 2016;21(5):1625-36. doi: 10.1590/1413-81232015215.19302015.

25. Bispo Júnior JP, Moreira DC. Núcleos de Apoio à Saúde da Família: concepções, implicações e desafios para o apoio matricial. Trab educ saúde. 2018;16(2):683-702. doi: 10.1590/1981-7746-sol00122.

26. Patrocínio SS da SM do, Machado CV, Fausto MCR. Núcleo de Apoio à Saúde da Família: proposta nacional e implementação em municípios do Rio de Janeiro. Saúde debate. 2015;39(spe):105-19. doi: 10.5935/01031104.2015S005373.

27. Martinez JFN, Silva MS, Silva AM. O Núcleo de Apoio à Saúde da Família em Goiânia (GO): percepções dos profissionais e gestores. Saúde debate. 2016;40(110):95-106. doi: 10.1590/0103-1104201611007.

28. Souza TT de, Knabben RJ, Calvo MCM. Caracterização de núcleos de apoio à saúde da família e integração às equipes de saúde da família vinculadas. Rev APS. 2017;20(4):551-64.

29. Oliveira GC, Schneider JF, Pinho LB, Camatta MW, Nasi C, Guimarães AN, Torres MEL. Matrix support in mental health in primary care: the vision of supporters and nurses. Rev gaúch. enferm. 2020;41(spe):e20190081. doi: 10.1590/1983-1447.2020.20190081.

30. Gurgel ALLG, Jorge MSB, Caminha ECCR, Maia Neto JP, Vasconcelos MGF. Mental health care in the family health strategy: the experience of matrix support. Rev enferm UERJ. 2017;25:1-6. doi: 10.12957/reuerj.2017.7101. 